Noname manuscript No.

(will be inserted by the editor)

\title{
On the effect of standard PFEM remeshing on volume conservation in free-surface fluid flow problems
}

\author{
Alessandro Franci · Massimiliano Cremonesi
}

Received: date / Accepted: date

\begin{abstract}
The aim of this work is to analyze the remeshing procedure used in the Particle Finite Element Method (PFEM) and to investigate how this operation may affect the numerical results. The PFEM remeshing algorithm combines the Delaunay triangulation and the Alpha Shape method to guarantee a good quality of the Lagrangian mesh also in large deformation processes. However, this strategy may lead to local variations of the topology that may cause an artificial change of the global volume. The issue of volume conservation is here studied in detail. An accurate description of all the situations that may induce a volume variation during the PFEM regeneration of the mesh is provided. Moreover, the crucial role of the parameter $\alpha$ used in the Alpha Shape method
\end{abstract}

\footnotetext{
A. Franci

International Center for Numerical Methods in Engineering (CIMNE), Universitat Politècnica de Catalunya (UPC), Gran Capitan s/n, 08034, Barcelona, Spain

Tel.: +34934016529

Fax: +34934016037

E-mail: falessandro@cimne.upc.edu

M. Cremonesi

Department of Civil and Environmental Engineering

Politecnico di Milano

Piazza Leonardo da Vinci 32, 20133 Milano, Italy
} 
is highlighted and a range of values of $\alpha$ for which the differences between the numerical results are negligible, is found. Furthermore, it is shown that the variation of volume induced by the remeshing reduces by refining the mesh. This check of convergence is of paramount importance for the reliability of the PFEM. The study is carried out for $2 \mathrm{D}$ free-surface fluid dynamics problems, however the conclusions can be extended to 3D and to all those problems characterized by significant variations of internal and external boundaries.

Keywords Particle Finite Element Method · volume conservation · alpha shape · remeshing

\section{Introduction}

The Particle Finite Element Method (PFEM) is a numerical strategy specifically designed for those problems where severe changes of topology occur, such as free surface fluid dynamics $[22,25,10,42$, 8], granular flows [43,4], non-linear solid mechanics [6], Fluid-Structure Interaction (FSI) [16, 19, $44,40]$, or thermal coupled problems $[1,30,37]$.

In the PFEM the mesh nodes are treated as particles and they move according to the Lagrangian equations of motion transporting their momentum together with all their physical properties. Nodes motion produces the deformation of the finite element mesh that needs to be rebuilt whenever a threshold value for the distortion is reached. The remeshing procedure is one of the most characteristic points of the PFEM [18]. This operation is performed via an efficient combination of the Delaunay tessellation [41,13] and the Alpha Shape (AS) method [12]. On the one hand, the Delaunay triangulation ensures the most homogeneous discretization for a given cloud of points. On the other hand, the AS technique allows the detection of the physical boundaries of the domain (free-surface contours, rigid boundaries or fluid-solid interfaces). The AS procedure performs an elemental control driven by a pre-defined parameter $\alpha$. Once the mesh is generated, the differential problem is integrated over the new mesh in the classical FEM fashion. 
The PFEM remeshing strategy guarantees a high level discretization creating the proper conditions for the accurate solution of the FEM problem at each time step. However, this operation has some drawbacks that may affect the numerical results. For instance, the perturbation of the equilibrium configuration caused by the elimination or the creation of elements, may worsen the quality and the convergence of the numerical solution. Furthermore, these local modifications may globally produce a variation of the volume of the computational domain.

The mentioned drawbacks of the PFEM remeshing can be seen as the price to pay in order to gain all the benefits that this Lagrangian technique can give. In [20] the advantages of the PFEM with respect to standard meshless methods have already been highlighted. In a more general sense, the PFEM is an extremely versatile strategy and it has been used to solve complex and challenging problems that other methods can handle with difficulty, as for example simulations of tunneling [7], forming processes [29] or melting of polymers [31]. In all these situations the PFEM has proved to cope excellently all the difficulties related to the complex geometries and/or to different physics.

The PFEM remeshing is here analyzed for free-surface incompressible fluid problems, but the findings of this work can be applied to any problem where major changes of external boundaries are encountered. The Lagrangian treatment of free-surface fluids is particularly critical due to the high distortions to which the Lagrangian mesh undergoes. In these cases, an extensive remeshing is required to ensure a good discretization at each computation step. Furthermore, for incompressible materials the issue of volume conservation is crucial. The conjunction of these two aspects makes free-surface fluid problems the ideal framework for testing the PFEM remeshing. In this work, the authors decided to focus on the 2D analysis essentially because it allows a better visualization of the mechanisms of volume variation associated to the PFEM remeshing than the 3D case. However, extension of the same ideas to three dimensional analysis is straightforward.

In the PFEM solution of incompressible fluids the mass conservation is affected by two different error sources, one associated to the numerical treatment of the governing equations and the other produced during the regeneration of the mesh [28]. The first mass variation source is experienced not 
only in PFEM but also in standard FEM. It may depend on various aspects as the iterative solver, the temporal discretization or the stabilization procedure. Several publications have already been dedicated to this crucial issue, as $[21,39,9,28]$. In this paper, this first source of volume variation is not analyzed and all the attention has been devoted to the second one because directly related to the PFEM remeshing procedure. The aim of this work is to study in detail the mechanisms that during the remeshing step may vary the global volume (thus the mass) and to check if it is possible to limit their effect. The final purpose is not a validation of the method (for which [25] or [24] can be seen), but a critical investigation of the PFEM remeshing procedure and the check of its convergence with respect to the mass conservation issue.

The paper is organized as follows. In the next section, the basic characteristics of the PFEM are presented. Then, the principal drawbacks of the PFEM are listed. In the following section, the issue of mass and volume conservation is analyzed and the typical mechanisms that induce a volume variation during the remeshing are described with the help of a numerical example. Finally, the dependence of the volume variation on the parameter $\alpha$ of the AS method is studied and the convergence of the method is verified on different examples.

\section{Essentials of the PFEM}

Basically, the PFEM can be described as a Lagrangian FEM in which the quality of the discretization is guaranteed at each time step by a remeshing strategy based on the Delaunay tessellation and the Alpha Shape method.

The first step of the method is the check of the mesh distortion. If the mesh used in the previous step is too distorted, all the simplices of the discretization are erased (Figures 1a and 1b). At this stage, the whole domain is represented by a cloud of points, or particles, that store all the information about physics, geometry, and kinematics (Figure 1b). Over this distribution of points the first trial mesh is generated using the Delauany triangulation (Figure 1c). In general, 
(a) Previous mesh

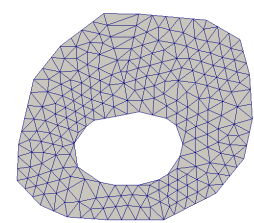

(c) Delaunay triangulation

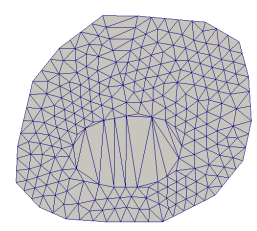

(b) Cloud of points

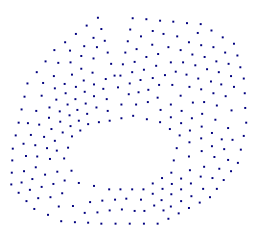

(d) New mesh

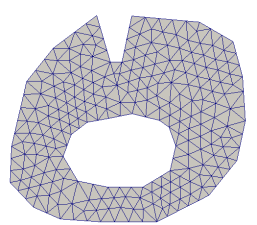

Fig. 1: Essential steps of the PFEM.

the Delaunay tessellation generates a convex figure which does not respect internal and external physical boundaries. The detection of the correct domain contours is performed by the AS technique which eliminates all those simplices that are excessively large or overly distorted (Figure 1d).

With the AS method each simplex built by the Delaunay triangulation is tested by comparing its circumradius $r_{e}$ to the mean mesh size $h$. The parameter $\alpha$ is used for establishing which elements are erased or not according to the following criterion

$$
\text { if } r_{e}>\alpha h \rightarrow \text { erase the } e-\text { element }
$$

After the Delaunay tesselation and the AS check, the new mesh with the correct (internal and external) boundaries can be used for the Finite Element solution. Note that at this point, the desired FEM strategy can be chosen. In fact, the PFEM does not impose any restriction on computational algorithms (e.g. time integration, unknown variables, solution scheme). Moreover, the same procedure applies for different physics (e.g. fluid mechanics, solid mechanics or thermal problems) without any distinction. 


\section{Drawbacks associated to PFEM remeshing}

If on the one hand the PFEM remeshing ensures a high quality of the FEM mesh also in large deformation problems, on the other hand it is associated to some drawbacks that require an accurate analysis.

First of all, the remeshing increases the computational time. In previous works it has been found that the time required for the remeshing depends almost linearly on the number of nodes but it grows slower than the time required for the solution of the discrete governing equations [27]. Moreover, the remeshing causes the loss of the connectivities of the previous discretization (see Figure 1b), consequently additional time is required to build the space operators defined by the differential problem (matrices and vectors).

Furthermore, the elimination of the elements of the previous mesh does not allow the storage of historical variables at element level. So that, for analyses in which the historical information is required (e.g. non-linear solid mechanics or computational plasticity), a data-transfer technique to recover element information must be added [5].

Another drawback is related to the boundary definition performed by the AS method. The check performed according to Eq.(1) highlights the crucial role of the parameter $\alpha$ in the PFEM remeshing algorithm. Different values of $\alpha$ may give different configurations, as shown in Figure 2. For $\alpha_{1}>\alpha_{2}$, the mesh obtained with $\alpha_{1}$ (Figure $2 \mathrm{~b}$ ) accepts a larger number of elements from the Delaunay discretization than the one given by $\alpha_{2}$ (Figure 2c), especially in the free-surface zone. On a extreme level, the mesh given by the Delaunay triangulation remains unchanged for extremely large values of $\alpha$. On the other hand, for excessive small values of $\alpha$, the AS method eliminates all the simplices created by the Delaunay tessellation. Specifically the lowest admissible $\alpha$ parameter is the one that corresponds to an equilateral triangle, $\alpha=1 / \sqrt{3}$. In $3 \mathrm{D}$ the minimum value for $\alpha$ is larger because, for a regular tetrahedron the ratio between the cirucmradius $\mathrm{r}$ and the minimum 
(a) Delaunay triangulation

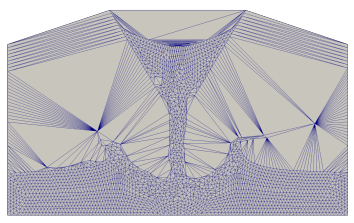

(b) Alpha Shape $\left(\alpha_{1}\right)$

(c) Alpha Shape $\left(\alpha_{2}\right)$
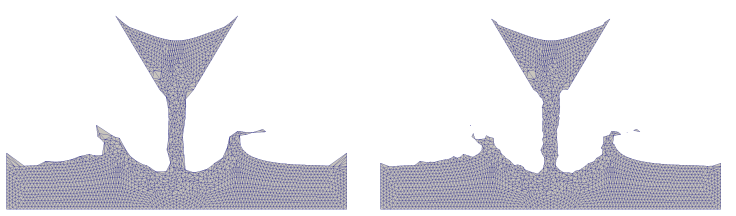

Fig. 2: Alpha Shape method applied over the same Delaunay triangulation $\left(\alpha_{1}>\alpha_{2}\right)$.

edge length is $\sqrt{3 / 8}[11]$. One of the objectives of this work is to verify whether there is a range of values of $\alpha$ for which the numerical results do not change significantly.

At global level, local changes of topology due to the AS scheme may produce the lack of preservation of the volume. In fact, if the sum of the volume of the erased elements is different than the one of the new simplices, the overall volume of the analysis domain is not conserved, leading also to possible mass oscillations.

The PFEM remeshing may also affect the solution accuracy because it induces non-physical modifications in the computational domains. The elimination of elements, the creation of new ones or just a change of connectivity, cause local perturbations of the equilibrium reached at the previous time step. Nodal values ensuring equilibrium on the previous mesh, may not guarantee equilibrium at the beginning of the new time step on the new discretization. These effects can produce a deterioration of the convergence or spurious oscillations of the pressure field.

This paper analyzes essentially the issue of volume conservation and the dependence on the parameter $\alpha$ while other drawbacks will be considered in future publications. 


\section{Analysis of mass conservation}

As introduced in section 1 , the study of mass conservation is performed on free-surface fluiddynamics problems. For a continuum, incompressible, homogeneous fluid filling the domain $V_{t}$, the equations of motion can thus be written as:

$$
\begin{array}{ll}
\rho \frac{D \mathbf{u}}{D t}=\nabla \cdot \boldsymbol{\sigma}+\rho \mathbf{b} & \text { in } V_{t} \times(0, T) \\
\nabla \cdot \mathbf{u}=0 & \text { in } V_{t} \times(0, T)
\end{array}
$$

where $\boldsymbol{u}=\boldsymbol{u}(\boldsymbol{x}, t)$ is the velocity, $\boldsymbol{\sigma}=\boldsymbol{\sigma}(\boldsymbol{x}, t)$ the Cauchy stress tensor, $\rho(\boldsymbol{x})$ is the fluid density, $\mathbf{b}(\boldsymbol{x}, t)$ the external body forces, $D /(D t)$ denotes the total time derivative and $(\nabla \cdot)$ is the divergence operator computed with respect to the current configuration $\boldsymbol{x}$. The problem (2)-(3) has to be supplemented with appropriate initial and boundary conditions and suitable constitutive equations.

A standard Galerkin finite element approach has been applied to discretize problem (2)-(3). A mixed velocity-pressure stabilized method proposed and validated in [28] is here applied. Details on the numerical formulation are out of the scope of this paper and can be found in [28]. Under the hypothesis of incompressible flow, density can be considered constant and mass variation is perfectly equivalent to volume variation [45]. Consequently, from now on, the study will be focused on volume variation.

Typically, two sources of volume variation can be identified: the first is related to the numerical solution of the governing equations and the second is associated to the remeshing technique.

Mass conservation equation is solved in standard finite element spirit and consequently, the inaccuracy of the numerical solver can affect the total mass conservation. The accuracy of the numerical solver depends on different parameters: the order of interpolations, the mean element size, the integration scheme and the time step increment. Moreover the original implementation of the PFEM for fluid-flow is based on equal-order interpolation scheme. So that, a stabilization technique should be used to guarantee the respect of LBB compatibility condition [3]. The stabilization terms have the effect of relaxing the incompressibility constrain and this may affect the mass preservation. 
Depending on the consistency of the stabilization procedure and the convergence capabilities of the method, the FEM solution may lead to a variation of the total mass and therefore of the total volume. The volume variation due to inaccuracy of the numerical scheme and due to the partial fulfillment of the incompressibility constrain is here called $\Delta V^{\text {num }}$.

Note that mass variation $\Delta V^{n u m}$ depends strongly on the numerical method and the stabilization chosen, and it can be experienced in all the approaches based on the weak imposition of the mass conservation equation [36]. The lack of mass preservation is particularly evident in Lagrangian framework, because volume variation is immediately observed [2], but it can occur in all the standard Eulerian or ALE approaches.

On the other hand, the second source of volume variation is connected to the remeshing technique used to recreate the connectivity between elements. From one step to another, the Delaunay triangulation coupled with the AS scheme can create new elements or remove others (see Section 2). At the end of the remeshing step a volume variation, here called $\Delta V^{r e m}$, can be observed. This source of volume variation is characteristic of the PFEM and is independent on the governing equations and the physics of the problem.

Thus, the total volume variation can be computed by summing up the two contributions previously described as

$$
\Delta V=\Delta V^{\text {num }}+\Delta V^{r e m}
$$

Figure 3 helps to understand how the two contributions are computed. Starting from volume $V^{n}$, the fluid solver is applied, subsequently mesh nodes are moved according to the velocities and a new volume is obtained $\bar{V}^{n+1}$. At this stage, if the mesh is too distorted, a remeshing technique is applied and a new volume $V^{n+1}$ is obtained. Hence, the two contributions are computed as follows

$$
\Delta V^{n u m}=\bar{V}^{n+1}-V^{n} \quad \Delta V^{r e m}=V^{n+1}-\bar{V}^{n+1}
$$

It is important to recall that the two sources of volume variation cannot be considered uncorrelated. The variation of volume generated by remeshing may affect the variation due to the numerical 


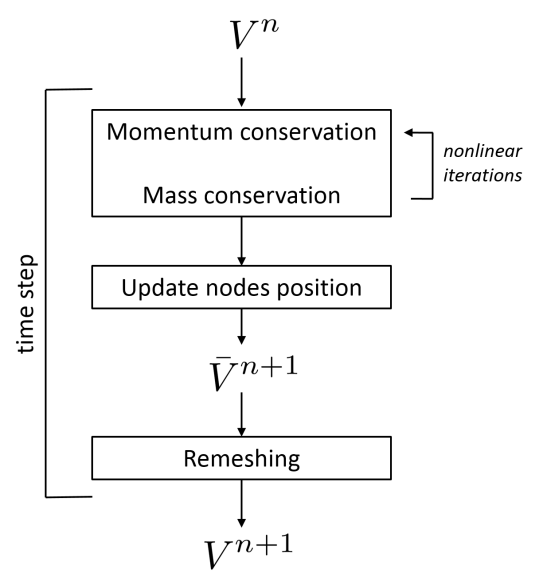

Fig. 3: Solution scheme for volume volumes calculation

scheme. In fact, the remeshing induces a perturbation of the equilibrium through the creation and elimination of elements, and consequently affects the fulfillment of the incompressibility constrain. Viceversa, the variation due to the inaccuracy of the solver, can lead to inaccurate position of the nodes and, therefore, influence the final volume of the domain.

Both sources of mass variation are important and must be seriously considered when an analysis of mass preservation is performed. However, the study of $\Delta V^{\text {num }}$ has not been included in this paper because it has been already presented in [28] and [15] for the same Lagrangian FIC-stabilized PFEM used in this work and all the attention has been devoted to the analysis of $\Delta V^{r e m}$.

4.1 Mechanisms of volume variation induced by remeshing

With the help of a numerical example, the typical mechanisms that induce volume variations are here described. A proper understanding of these situations is essential in order to design the right countermeasures to reduce their effects. The collapse of a water column against a rigid obstacle presented in [17] has been chosen as reference problem, because it induces all the typical situations of volume variation caused by the remeshing. The initial geometry and problem data are given in Figure 4 and Table 1. 


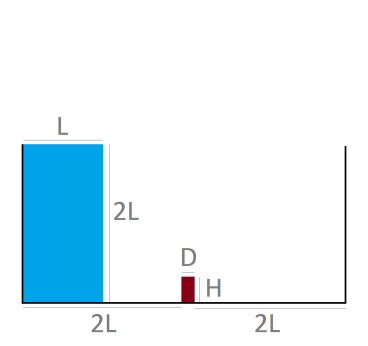

Fig. 4: Dam break against a rigid step. Initial geometry.

\begin{tabular}{|c|c|}
\hline $\mathrm{L}$ & $0.146 \mathrm{~m}$ \\
$\mathrm{H}$ & $0.048 \mathrm{~m}$ \\
$\mathrm{D}$ & $0.024 \mathrm{~m}$ \\
viscosity & $10^{-3} \mathrm{~Pa} \cdot \mathrm{s}$ \\
density & $10^{3} \mathrm{~kg} / \mathrm{m}^{3}$ \\
\hline
\end{tabular}

Table 1: Dam break against a rigid step. Problem data.

During the advancement of the water column two mechanisms of volume variation are recognizable. If no slip conditions are considered, the only way the flow can advance is by creating a new element composed by the nearest free-surface node and the next wall particle, as shown in Figures $5 \mathrm{a}$ and $5 \mathrm{~b}$. The area of the new elements, highlighted in Figure 5b, represents an increase of total volume induced by the remeshing.

(a) Before meshing

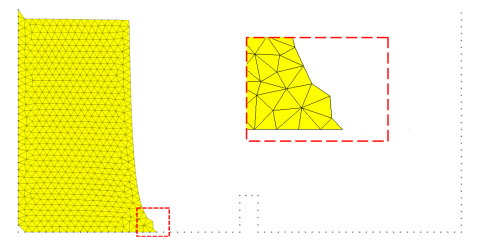

(b) After meshing

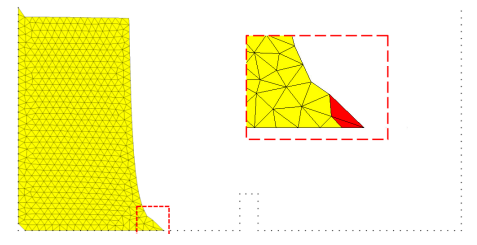

Fig. 5: Mechanism of volume gain due to remeshing. Advance of the wave front. 
While the wave front is advancing, the height of the water column is decreasing. This dual situation produces the elimination via the AS of those elements composed by the free-surface nodes located at the top of the column and the highest wall particles (see Figures 6a, 6b).

(a) Before meshing

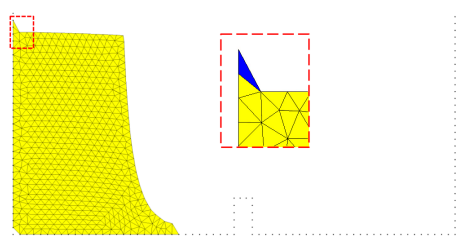

(b) After meshing

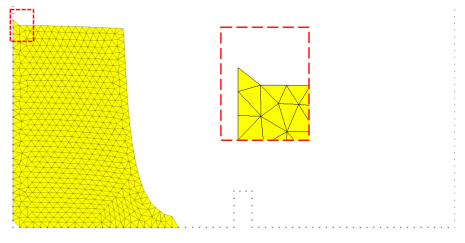

Fig. 6: Mechanism of volume loss due to remeshing. Recede of a fluid volume.

The elongation of the free-surface boundaries may also represent a source of volume variation. In fact, if the mesh is not refined enough, some boundary elements may not fulfil the AS criterion due to the excessive stretching of their free-surface edge. The elimination of those elements causes the formation of artificial waves at the free-surface, as shown in Figure 7.

Fluid drops can also produce a variation of the total volume. In particular, when a particle separates from the domain, an element is removed (Figures $8 \mathrm{a}, 8 \mathrm{~b}$ ). On the contrary, some new elements are created to allow the reinsertion of a particle which comes close enough to the bulk. (Figures 9a, 9b). Note that the same applies also to the detachment and reinsertion of a group of elements.

In the PFEM, the definition of the contact surfaces is automatically performed by the AS technique and in general, can lead to a volume increase. Examples are given in the contact with rigid 
(a) Before meshing

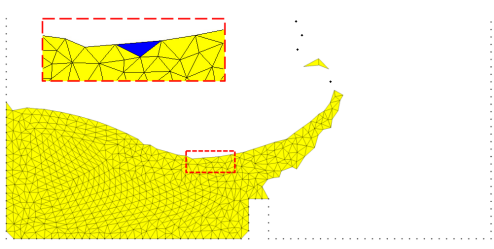

(b) After meshing

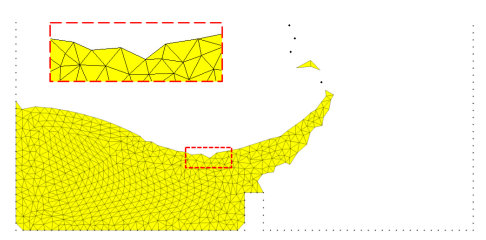

Fig. 7: Mechanism of volume loss due to remeshing. Formation of artificial waves.

(a) Before meshing

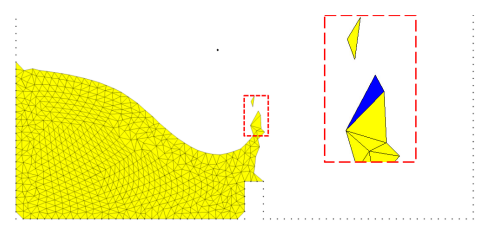

(b) After meshing

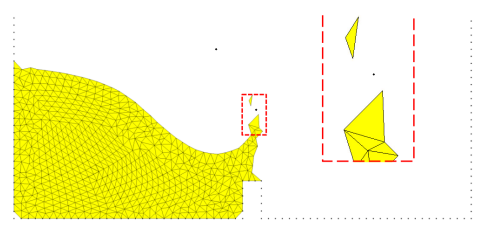

Fig. 8: Mechanism of volume loss due to remeshing. Formation of a free particle.

walls (see Figure 10), the contact between two fluid streams or when two free-surface boundaries are joined (Figure 11).

Note that all the mechanisms of volume variation presented for this $2 \mathrm{D}$ problem have their correspondent mechanism in 3D. For example, in Figure 12 the same mechanism presented for the 2D case in Figure 10 is provided. 
(a) Before meshing

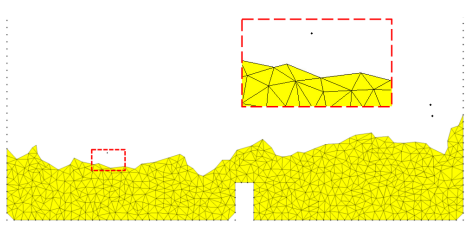

(b) After meshing

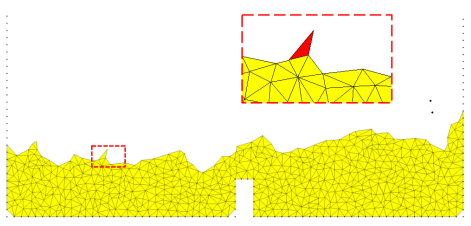

Fig. 9: Mechanism of volume gain due to remeshing. Free particle reinsertion.

(a) Before meshing

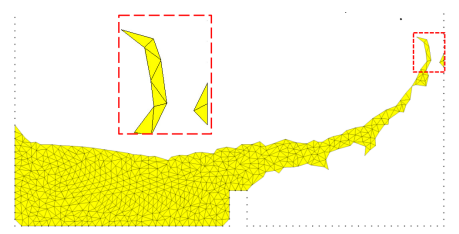

(b) After meshing

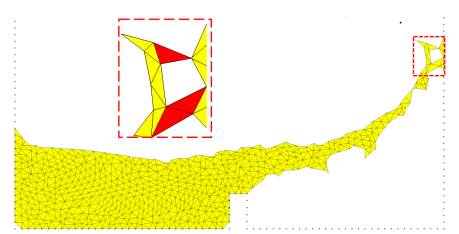

Fig. 10: Mechanism of volume gain due to remeshing. Impact of a fluid stream with a boundary.

To better appreciate the creation of the new elements on the solid boundaries, the central section of the 3D domain of Figure 12 is represented in Figure 13 highlighting in red the elements created by the $3 \mathrm{D}$ remeshing.

All these situations are intrinsically connected to the method and cannot be completely removed, in $2 \mathrm{D}$ as in 3D. However there exist some ad hoc strategies that can be used to reduce their effects. For instance, the use of a penalized parameter $\alpha$ for the free-surface elements [14], a local refinement of the free boundaries, or a specific treatment of the contact elements [38]. Nevertheless the aim 
(a) Before meshing

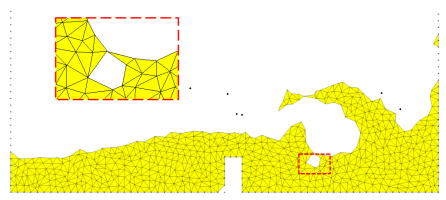

(b) After meshing

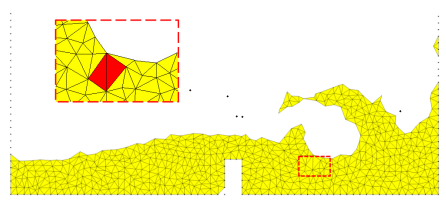

Fig. 11: Mechanism of volume gain due to remeshing. Union of free-surface boundaries.

(a) Before meshing

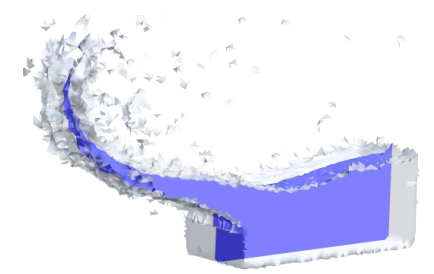

(b) After meshing

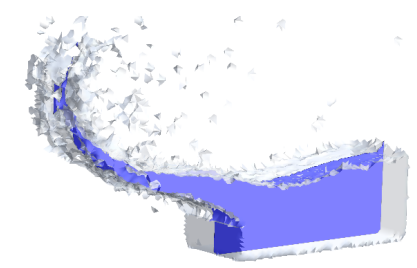

Fig. 12: 3D mechanism of volume gain due to remeshing. Impact of a fluid stream with a boundary.

of this work is to analyze the PFEM in its general and standard formulation. Hence, these local strategies will not be taken into consideration. Despite this, it will be shown that just the use of a proper values for the parameter $\alpha$ and the mesh refinement can be enough to control and keep limited the volume variation due to the PFEM remeshing. 
(a) Before meshing

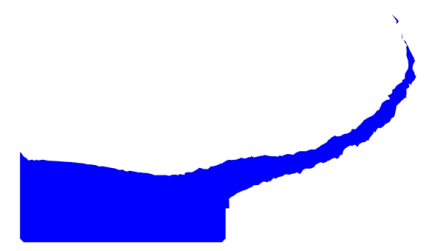

(b) After meshing

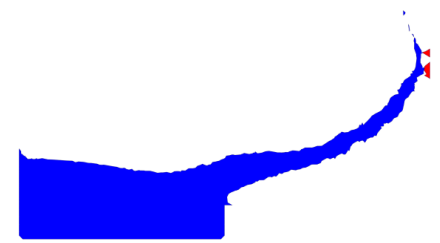

Fig. 13: 3D mechanism of volume gain due to remeshing. Impact of a fluid stream with a boundary, central section of Fig. 12.

\section{Numerical examples}

In this section, four numerical examples are studied to find a range of values of $\alpha$ for which the volume variation induced by the PFEM remeshing is limited and the numerical results do not change too much. Moreover, the convergence of the method with respect to the mesh size is tested. First, the same dam break with obstacle of Figure 4 is analyzed to show the dependence of the volume conservation on $\alpha$ and to select a good value of $\alpha$ for the volume conservation issue. With this value of $\alpha$, the convergence of the PFEM with respect to the mesh size in terms of volume conservation is studied for a standard dam break, for an impact of a fluid volume against a rigid wall and for the mixing of a viscous fluid.

All the numerical examples are studied using an uniform mesh. However, Eq.(1) shows that the extension to hetereogeneous meshes is straightforward. The only complication associated to these cases is that the element size $h$ is not fixed but it must be computed locally. 
5.1 Water dam break against a rigid step

The collapse of a water column against a rigid step (Figure 4) has been solved for different values of $\alpha$ and with a fixed mean mesh size $h=0.005 \mathrm{~m}$. The total duration of the analysis is $2 s$ and a time step $\Delta t=0.0005 \mathrm{~s}$ has been used.

In Figure 14 the results of the numerical simulations are compared to laboratory tests [17] for three different values of $\alpha$ and for two time instants $(t=0.2 s$ and $t=0.3 \mathrm{~s})$. These plots show that for each $\alpha$ a different solution is obtained confirming that the choice of $\alpha$ affects the numerical results. However, the differences are small and the dynamics of the example is respected despite the use of a large range of $\alpha$ (from 1.05 to 1.6 ).

Nevertheless, after the impact against the rigid boundaries and the consequent formation of splashes, the results given by different $\alpha$ start to diverge and, in particular, for large values of $\alpha$ the resulting volume variation is extremely high. Figure 15 shows the volume variation $\Delta V^{r e m}$ obtained at the end of the analysis $(t=2.0 s)$ for different values of $\alpha$. The graph shows that the conjunction of an analysis dominated by splashes and impacts against the boundaries with the use of large values of $\alpha$ on a relatively coarse mesh, may induce a huge increase of volume. From the graph an almost linear dependence between $\alpha$ and $\Delta V^{r e m}$ can be deduced. For $\alpha \leq 1.2$, the final balance of volume variation is negative; for $\alpha>1.2$ is positive. For values of $\alpha$ between $\alpha=1.15$ and $\alpha=1.25$, the absolute value curve has a quite flat minimum zone for which $\Delta V^{r e m}$ is lower than the $10 \%$. In particular, for this problem the minimum $\left(\left|\Delta V^{r e m}\right| \approx 1 \%\right)$ is reached for $\alpha=1.2$. Observe that this value is close to the values suggested in previous works $[23,27,32,35]$ according to empirical considerations only.

It must be clarified that is not possible to determine universally the best value for $\alpha$, a different example may give a slight different minimum value, and hence this cannot be among the objectives of the present study. However, it can be shown that, although the results depend on $\alpha$, there exists a range of values of $\alpha$ for which the numerical results do not change significantly. 
(a) $t=0.2 \mathrm{~s}$, experimental (b) $t=0.3 \mathrm{~s}$, experimental
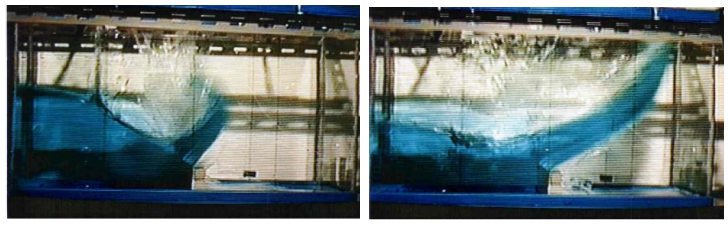

(c) $t=0.2 s, \alpha=1.05$

(d) $t=0.3 s, \alpha=1.05$

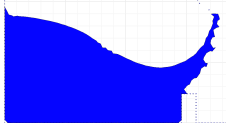

(e) $t=0.2 s, \alpha=1.20$

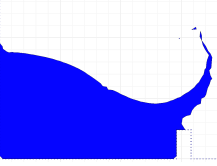

(g) $t=0.2 s, \alpha=1.60$

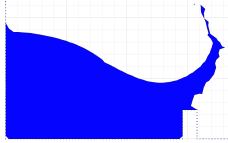

(f) $t=0.3 s, \alpha=1.20$

(h) $t=0.3 s, \alpha=1.60$
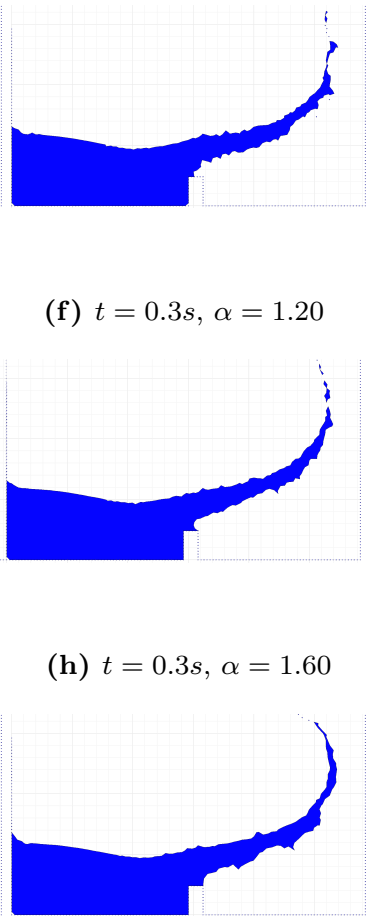

Fig. 14: Water dam break against a rigid step. Experimental [17] and numerical results for three different values of $\alpha$ at $t=0.2 s$ and $t=0.3 s$.

Note also that the numerical example here solved is particularly critical for the issue of volume conservation. In simpler problems (e.g. a steady fluid motion or at least with less splashes) the range of acceptable values for $\alpha$ will be larger than the one found for this case. 


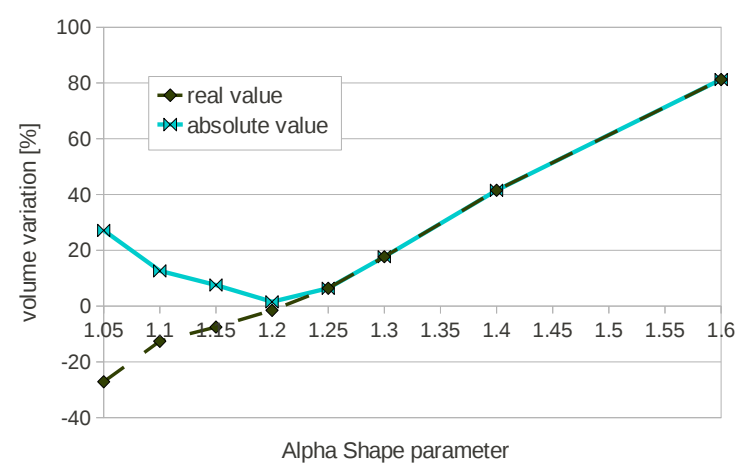

Fig. 15: Water dam break against a rigid step. Volume variation for different values of $\alpha$ at $t=2.0 \mathrm{~s}$.

5.2 Water dam break

The collapse of a water column on a rigid horizontal plane [26] is here studied varying the mesh size and keeping fixed the value of $\alpha=1.2$. The initial configuration and the problem data are provided in Figure 16 and Table 2, respectively.

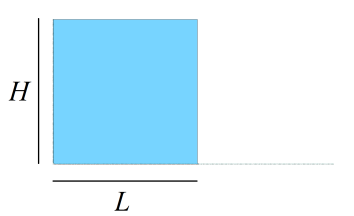

Fig. 16: Water dam break. Initial geometry.

\begin{tabular}{|c|c|}
\hline$H$ & $0.05715 \mathrm{~m}$ \\
$L$ & $0.05715 \mathrm{~m}$ \\
viscosity & $10^{-3} \mathrm{~Pa} \cdot \mathrm{s}$ \\
density & $10^{3} \mathrm{~kg} / \mathrm{m}^{3}$ \\
\hline
\end{tabular}

Table 2: Water dam break. Problem data.

This problem is useful to understand the phenomena occurring during the advance of a fluid front over rigid boundaries in the PFEM. In this test the increase of volume is exclusively produced by the mechanism of the wave advancing (Figure 5) while the loss of volume is given by the mechanisms of wave retirement (Figure 6) and by the formation of artificial waves (Figure 7).

Figure 17 shows the fluid configurations at $t=0.15 \mathrm{~s}$ obtained with the coarsest $(h=0.006 \mathrm{~m})$ and the finest $(h=0.001 \mathrm{~m})$ meshes. 
(a) Coarsest mesh

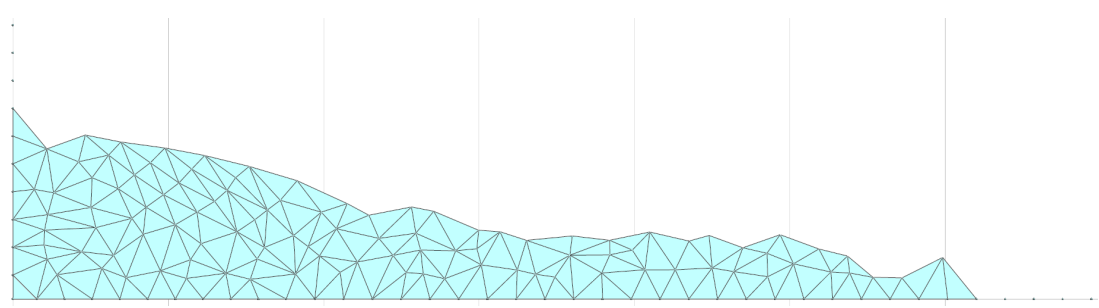

(b) Finest mesh

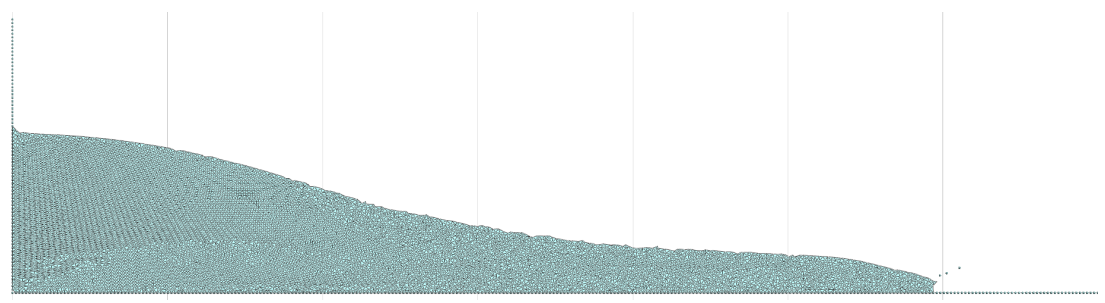

Fig. 17: Water dam break. Deformed configurations at $t=0.15 \mathrm{~s}$ obtained with the coarsest and the finest tested meshes and $\alpha=1.2$.

The graph of Figure 18 plots the time evolution of volume variation due to remeshing obtained using the coarsest and the finest meshes.

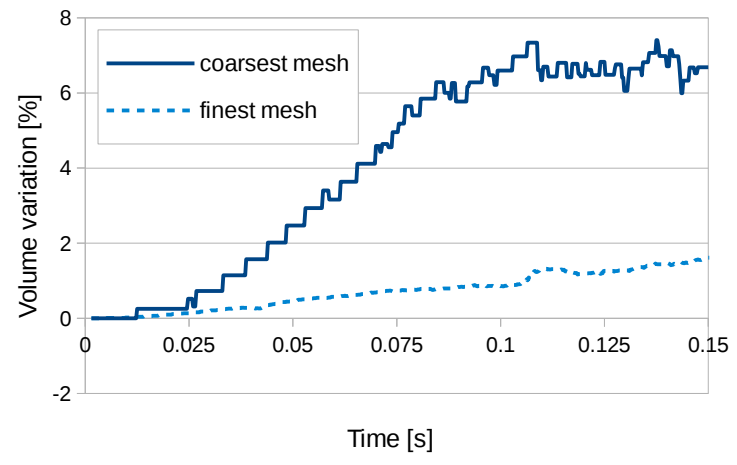

Fig. 18: Water dam break. Time evolution of volume variation due to remeshing obtained with the coarsest and the finest tested meshes and $\alpha=1.2$. 
The graphs show that for both meshes the tendency is to increase the volume, however a clear reduction of volume gain is obtained by reducing the mesh size.

For better visualizing the convergence in terms of mass conservation, in Figure 19 the volume variation is plotted for all the tested meshes separating the contribution of remeshing to volume gain (wave advancing) and volume loss (wave retirement). In particular, the dashed curve of Figure 19 represents the sum of only the positive variations of volume $\left(\sum \Delta V^{r e m}\right.$, if $\left.\Delta V^{r e m}>0\right)$ while the continuous curve refers to the sum of all the negative ones $\left(\sum \Delta V^{r e m}\right.$, if $\left.\Delta V^{r e m}<0\right)$.

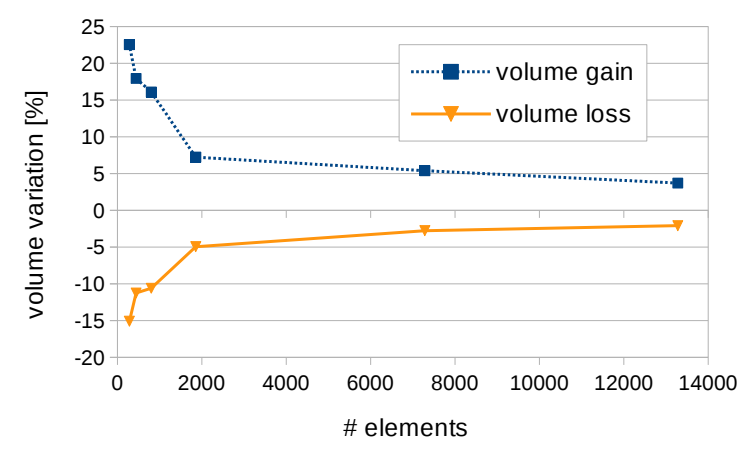

Fig. 19: Water dam break. Accumulated volume variation after $0.15 \mathrm{~s}$ obtained for all the tested discretizations and $\alpha=1.2$.

The graphs show a clear convergence in terms of volume preservation. Both volume losses and gains, that are due to different volume variation mechanisms, reduce progressively with the mesh refinement. The graphs of Figure 19 also confirm the conclusion drawn from Figure 18. In fact, the volume gains are larger than the volume losses for all the tested meshes.

In conclusion, from this study it can be deduced that in the initial phase of a dam break, the PFEM remeshing induces an overall increment of volume, although this increase reduces by refining the mesh. This means that the mechanism of volume gain of Figure 5 is prevalent with respect to the ones responsible of volume loss (see Figures 6 and 7), also for fine meshes. 
5.3 Impact of a viscous fluid against a rigid wall

In this test, a mass of viscous fluid falls into a fixed container. The initial geometry and the problem data are provided in Figure 20 and Table 3, respectively.

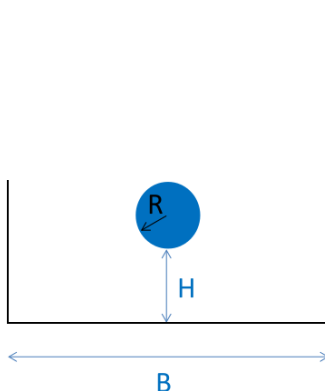

Fig. 20: Impact of a viscous fluid against a rigid wall. Initial geometry.

\begin{tabular}{|c|c|}
\hline$H$ & $0.07 \mathrm{~m}$ \\
$B$ & $0.45 \mathrm{~m}$ \\
$R$ & $0.025 \mathrm{~m}$ \\
viscosity & $10^{-1} \mathrm{~Pa} \cdot \mathrm{s}$ \\
density & $10^{3} \mathrm{~kg} / \mathrm{m}^{3}$ \\
\hline
\end{tabular}

Table 3: Impact of a viscous fluid against a rigid wall. Problem data.

This example has been conceived to better understand the interaction with walls (see Figure 10). In this test, the fluid mass, subjected only to the gravity force, falls down and collides with the horizontal wall. When the fluid mass comes close enough to the boundary, some elements are built between the fluid surface and the rigid wall. These new elements (highlighted in red in Figure 21) can be considered as contact elements and they are responsible for the total volume variation. However, differently from the contact domain method $[33,34]$, in which these elements are employed to detect the contact surfaces and to compute contact forces, in this case they are treated as standard fluid elements (with a physical meaning and the same constitutive relation of the fluid material).

The problem has been solved for a fixed value of $\alpha=1.2$ and for three different mesh sizes. Figure 22 shows the volume variation for the three meshes. The final net increase of the volume depends only on the elements created to manage the contact and, as expected, decreases reducing 


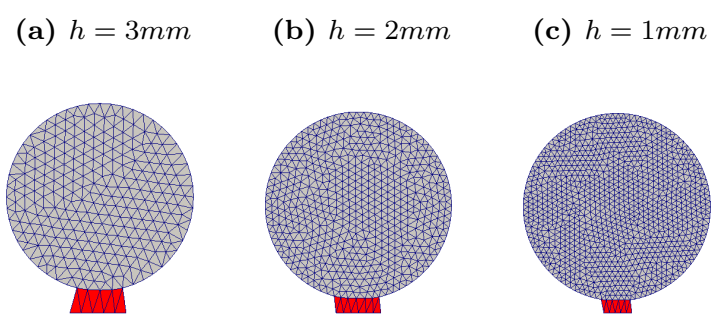

Fig. 21: Impact of a viscous fluid against a rigid wall. Snapshots at the contact instant for the three meshes

the mean mesh size. As in the previous example, a clear convergence can be observed, showing that mass variation due to surface contact can be controlled by reducing the typical mesh size.

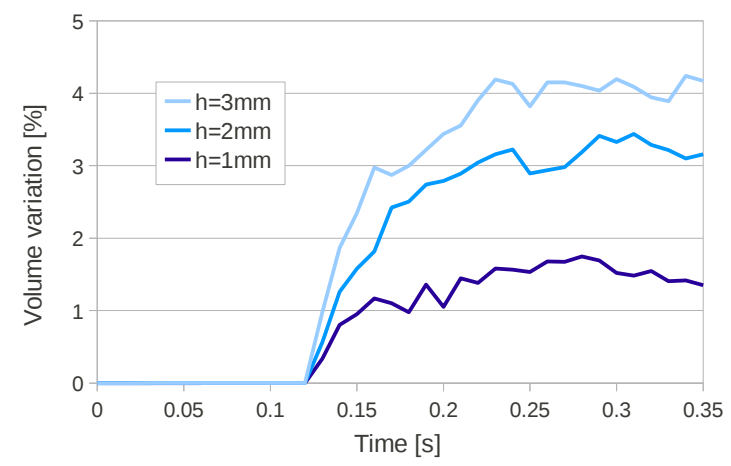

Fig. 22: Impact of a viscous fluid against a rigid wall. Volume variation on time for different mesh sizes.

It must also be observed that the typical dimension of contact elements depends also on the parameter $\alpha$. Larger values of alpha generate larger contact elements. Figures 23 shows the comparison of the contact instant for three values of alpha, where no significant differences can be observed. Table 4 contains the final volume variation varying alpha for $h=3 \mathrm{~mm}$. It can be noticed that the alpha parameter does not affect significantly the total mass in this particular situation.

\subsection{Mixing of a viscous fluid}

In this test, a volume of viscous fluid falls into a tank filled with the same fluid. The initial geometry and the problem data are given in Figure 24 and Table 5, respectively. 


\begin{tabular}{|c|c|}
\hline alpha & Volume variation \\
\hline 1.1 & $4.02 \%$ \\
\hline 1.2 & $4.31 \%$ \\
\hline 1.4 & $4.36 \%$ \\
\hline
\end{tabular}

Table 4: Impact of a viscous fluid against a rigid wall. Volume variation due to the alpha parameter.
(a) $\alpha=1.1$
(b) $\alpha=1.2$
(c) $\alpha=1.4$
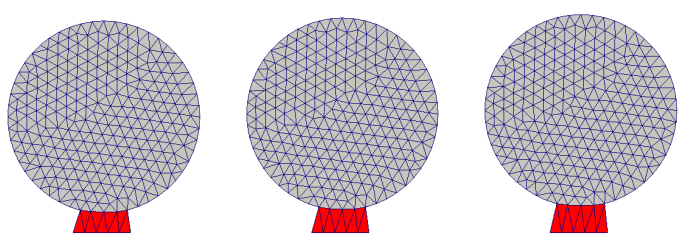

Fig. 23: Impact of a viscous fluid against a rigid wall. Snapshots at the contact instant for the three different values of the parameter $\alpha$.

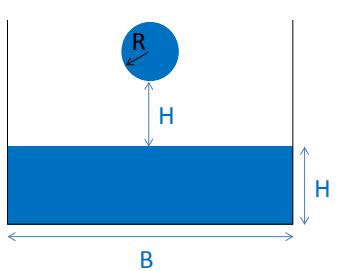

Fig. 24: Mixing of a viscous fluid. Initial geometry.

\begin{tabular}{|c|c|}
\hline$H$ & $0.07 \mathrm{~m}$ \\
$B$ & $0.45 \mathrm{~m}$ \\
$R$ & $0.025 \mathrm{~m}$ \\
viscosity & $10^{-1} \mathrm{~Pa} \cdot \mathrm{s}$ \\
density & $10^{3} \mathrm{~kg} / \mathrm{m}^{3}$ \\
\hline
\end{tabular}

Table 5: Mixing of a viscous fluid. Problem data.

As in the previous examples, the problem is solved for $\alpha=1.2$ and for different mesh sizes (from $h=0.005 m$ to $h=0.0015 m$ ). This example is mainly characterized by the mechanisms of Figure 10 and 11. However, differently from the first example, in this problem the amount of splashes is reduced due to the high viscosity of the fluid considered. This facilitates the study of the PFEM remeshing procedure. 

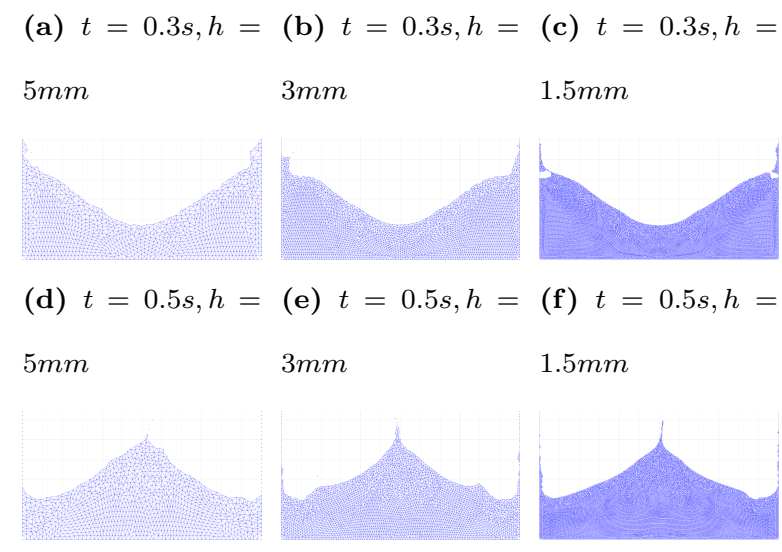

(g) $t=1.05 \mathrm{~s}, h=$ (h) $t=1.05 \mathrm{~s}, h=$ (i) $t=1.05 \mathrm{~s}, h=$

$5 \mathrm{~mm} \quad 3 \mathrm{~mm} \quad 1.5 \mathrm{~mm}$

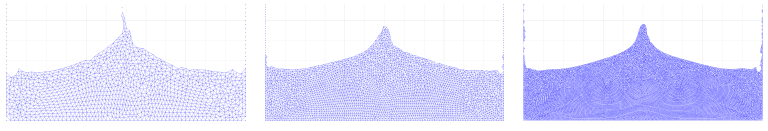

Fig. 25: Mixing of a viscous fluid. Results for different meshes at three time instants $(\alpha=1.2)$.

Figure 25 shows that the dynamics of the problem is well reproduced also for a coarse mesh. The curves of Figure 26 are the volume variation on time obtained for four different meshes. The results

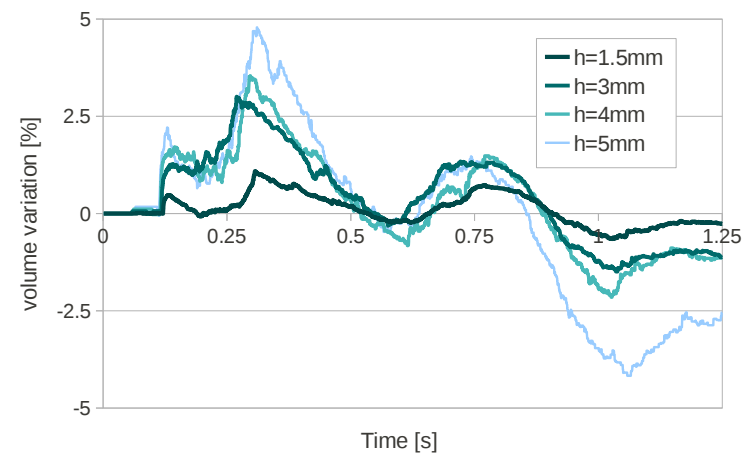

Fig. 26: Mixing of a viscous fluid. Volume variation on time for different mesh sizes and $\alpha=1.2$.

confirm quantitatively the convergence tendency deducible from the previous pictures, showing that volume variation tends to zero by reducing the size of the mesh. 
In Figure 27 the numerical results obtained for three different values of $\alpha$ at the end of the analysis $(t=1.5 \mathrm{~s})$ are given. The pressure contours (negative values for the compression) are also plotted. The three results are almost identical despite the use of different values of $\alpha$ and this

(a) $\alpha=1.15$

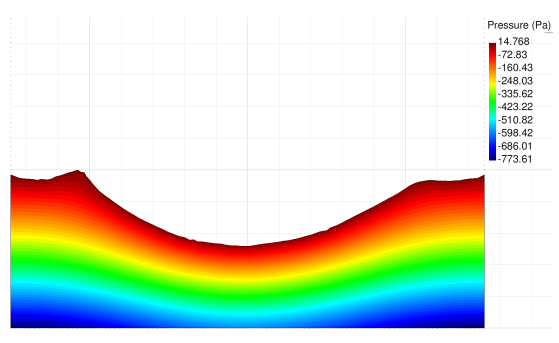

(b) $\alpha=1.20$

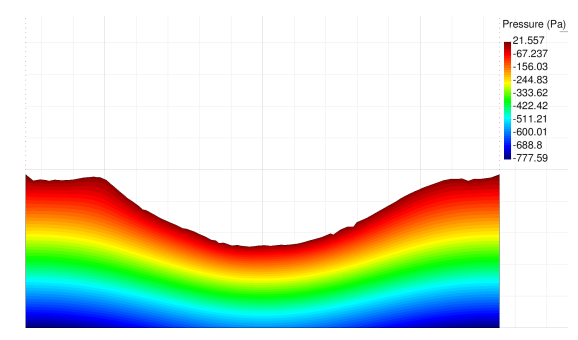

(c) $\alpha=1.25$

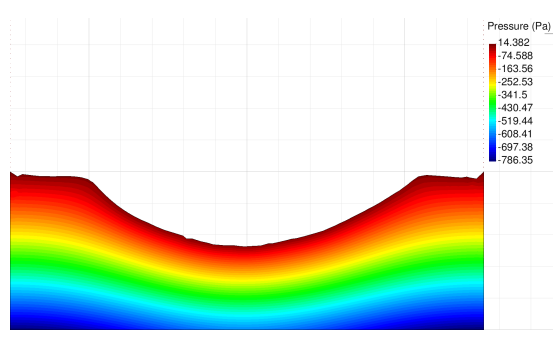

Fig. 27: Mixing of a viscous fluid. Results at $t=1.5 \mathrm{~s}$ for different values of $\alpha$.

confirms that the role of the parameter $\alpha$ is less crucial in this problem. 


\section{Conclusions}

This paper has been devoted to study the Particle Finite Element Method remeshing procedure and to analyze how this operation may affect the numerical results. One of the key features of the PFEM is the efficient solution of problems with severe changes of the topology. Delaunay triangulation coupled with the Alpha Shape method allows for a fast generation of a new mesh and for a robust identification of the internal and external boundaries. However, this remeshing strategy may lead to undesired topological modifications and to an overall volume variation.

The definition of the typical mechanisms that induce these volume changes in the PFEM simulations is crucial to design the optimum countermeasures. For this reason, in the first part of the paper all these mechanisms have been described and graphically illustrated. Their identification can definitely be helpful to anyone wishing to carry out an analysis with PFEM in the future. A free-surface benchmark problem, as the water dam break against a rigid obstacle, has been used to show how each one of these mechanisms may affect a different phase of the analysis and induce volume variations. The principal mechanisms have been clearly highlighted also for the rest of numerical tests.

As expected, all the examples studied in this work confirm that there is a clear dependence between the parameter $\alpha$ of the Alpha Shape method and $\Delta V^{r e m}$. It has been shown that for values close to $\alpha=1.2$, the numerical results do not change significantly and the variation of volume is acceptable also for problems involving highly unsteady flows. The range of $\alpha$ proposed in this work confirms the values used in previous papers (e.g. $[22,23,27,32,35])$.

From the analysis of the collapse of a water column, it has been found that the PFEM remeshing induces an increment of volume during the first phase of the dam break, for all the tested meshes. However, this volume variation reduces progressively by refining the mesh. The convergence of the PFEM remeshing in terms of volume preservation has been verified also for all the other tests. These results show that, despite the continuous remeshing, the PFEM works in the spirit of the 
finite element ensuring better results when the mesh size is reduced, at least in terms of volume conservation.

In conclusion, it has been shown that, although the lack of volume preservation is a intrinsic drawback of the PFEM remeshing, it can be limited by using a proper value of $\alpha$ and moreover it can be controlled and strongly reduced by refining the mesh.

\section{References}

1. R. Aubry, S. R. Idelsohn, and E. Oñate. Particle finite element method in fluid-mechanics including thermal convection-diffusion. Computers and Structures, 83:1459-1475, 2005.

2. R. Aubry, E. Oñate, and S. R. Idelsohn. Fractional Step Like Schemes for Free Surface Problems with Thermal Coupling Using the Lagrangian PFEM. Computational Mechanics, 38(4-5):294-309, apr 2006.

3. F. Brezzi. On the existence, uniqueness and approximation of saddle-point problems arising from lagrange multipliers. Revue française d'automatique, informatique, recherche opérationnelle. Série rouge. Analyse numérique, 8(R-2):129-151, 1974.

4. J. Cante, C. Davalos, J. A. Hernandez, J. Oliver, P. Jonsen, G. Gustafsson, and H. Haggblad. Pfem-based modeling of industrial granular flows. Computational Particle Mechanics, 1 (1):47-70, 2014.

5. J. M. Carbonell. Doctoral thesis: Modeling of Ground Excavation with the Particle Finite Element Method. 2009.

6. J. M. Carbonell, E. Oñate, and B. Suarez. Modeling of ground excavation with the particle finite-element method. Journal of Engineering Mechanics, 136:455-463, 2010.

7. J. M. Carbonell, E. Oñate, and B. Suarez. Modelling of tunnelling processes and cutting tool wear with the particle finite element method (pfem). Computational Mechanics, 52 (3):607-629, 2013.

8. M. Cremonesi, L. Ferrara, A. Frangi, and U. Perego. Simulation of the flow of fresh cement suspensions by a lagrangian finite element approach. Journal of Non-Newtonian Fluid Mechanics, 165:1555-1563, 2010.

9. M. Cremonesi, A. Frangi, and U. Perego. A lagrangian finite element approach for the analysis of fluid-structure interaction problems. International Journal of Numerical Methods in Engineering, 84:610630, 2010.

10. M. Cremonesi, A. Frangi, and U. Perego. A lagrangian finite element approach for the simulation of waterwaves induced by landslides. Computer and Structures, 89:1086-1093, 2011.

11. A. N. Tornberg E. VanderZee and D.Guoy. Triangulation of simple 3d shapes with well-centred tetrahedra. Proceedings of the 17th International Meshing Roundtable, pages 19-35, 2008. 
12. H. Edelsbrunner and E.P. Mucke. Three dimensional alpha shapes. ACM Trans Graphics, 13:43-72, 1999.

13. H. Edelsbrunner and T.S. Tan. An upper bound for conforming delaunay triangulations. Discrete and Computational Geometry, 10 (2):197:213, 1993.

14. A. Franci. Unified Lagrangian formulation for fluid and solid mechanics, fluid-structure interaction and coupled thermal problems using the PFEM. Doctoral Thesis, Universitat Politècnica de Catalunya, 2015.

15. A. Franci, E. Oñate, and J. M. Carbonell. On the effect of the bulk tangent matrix in partitioned solution schemes for nearly incompressible fluids. International Journal for Numerical Methods in Engineering, $102: 257-277,2015$.

16. A. Franci, E. Oñate, and J. M. Carbonell. Unified lagrangian formulation for solid and fluid mechanics and fsi problems. Computer Methods in Applied Mechanics and Engineering, 298:520-547, 2016.

17. D.M. Greaves. Simulation of viscous water column collapse using adapting hierarchical grids. International Journal of Numerical Methods in Engineering, 50:693-711, 2006.

18. S. R. Idelsohn, N. Calvo, and E. Oñate. Polyhedrization of an arbitrary point set. Computer Methods in Applied Mechanics and Engineering, 92 (22-24):2649-2668, 2003.

19. S. R. Idelsohn, J. Marti, A. Limache, and E. Oñate. Unified lagrangian formulation for elastic solids and incompressible fluids: Applications to fluid-structure interaction problems via the pfem. Computer Methods In Applied Mechanics And Engineering, 197:1762-1776, 2008.

20. S. R. Idelsohn and E. Oñate. To mesh or not to mesh. that is the question... Computer Methods in Applied Mechanics and Engineering, 195:4681-4696, 2006.

21. S. R. Idelsohn and E. Oñate. The challenge of mass conservation in the solution of free surface flows with the fractional step method. problems and solutions. Communications in Numerical Methods in Engineering, 26(10):1313-1330, 2008.

22. S. R. Idelsohn, E. Oñate, and F. Del Pin. The particle finite element method: a powerful tool to solve incompressible flows with free-surfaces and breaking waves. International Journal for Numerical Methods in Engineering, 61:964-989, 2004.

23. S. R. Idelsohn, E. Oñate, F. Del Pin, and N. Calvo. Fluid-structure interaction using the particle finite element method. Computer methods in applied mechanics and engineering, 195:2100-2113, 2006.

24. S. R. Idelsohn, M. A. Storti, and E. Oñate. Lagrangian formulations to solve free surface incompressible inviscid fluid flows. Computer Methods in Applied Mechanics and Engineering, 191, 6-7:583-593, 2001.

25. A. Larese, R. Rossi, E. Oñate, and S. R. Idelsohn. Validation of the particle finite element method (pfem) for simulation of free surface flows. International Journal for Computer-Aided Engineering and Software, $25: 385-425,2008$. 
26. J. C. Martin and W.J. Moyce. Part iv. an experimental study of the collapse of liquid columns on a rigid horizontal plane. Philosophical Transactions of the Royal Society of London. Series A, Mathematical and Physical Sciences, 244 (882):312-324, 1952

27. E. Oñate, M. A. Celigueta, S. R. Idelsohn, F. Salazar, and B. Suarez. Possibilities of the particle finite element method for fluid-soil-structure interaction problems. Computation mechanics, 48:307-318, 2011.

28. E. Oñate, A. Franci, and J. M. Carbonell. Lagrangian formulation for finite element analysis of quasiincompressible fluids with reduced mass losses. International Journal for Numerical Methods in Fluids, 74 (10):699-731, 2014.

29. E. Oñate, A. Franci, and J. M. Carbonell. A particle finite element method for analysis of industrial forming processes. Computational Mechanics, 54:85-107, 2014.

30. E. Oñate, J. Marti, R. Rossi, and S. R. Idelsohn. Analysis of the melting, burning and flame spread of polymers with the particle finite element method. Computer Assisted Methods in Engineering and Science, $20: 165-184,2013$.

31. E. Oñate, R. Rossi, S. R. Idelsohn, and K. Butler. Melting and spread of polymers in fire with the particle finite element method. International Journal of Numerical Methods in Engineering, 81 (8):1046-1072, 2010.

32. Eugenio Oñate, Sergio R. Idelsohn, Miguel A. Celigueta, and Riccardo Rossi. Advances in the particle finite element method for the analysis of fluid-multibody interaction and bed erosion in free surface flows. Computer Methods in Applied Mechanics and Engineering, 197(19-20):1777 - 1800, 2008. Computational Methods in Fluid-Structure Interaction.

33. X. Oliver, J. C. Cante, R. Weyler, C. González, and J. Hernández. Particle finite element methods in solid mechanics problems. In: Oñate E, Owen R (Eds) Computational Plasticity. Springer, Berlin, 2007.

34. X. Oliver, S. Hartmann, J. C. Cante, R. Weyler, and J. Hernández. A contact domain method for large deformation frictional contact problems. part 1: theoretical basis. Computer methods in applied mechanics and engineering, 198:2591-2606, 2009.

35. E. Oñate, S. R. Idelsohn, M. A. Celigueta, R. Rossi, J. Marti, J. M. Carbonell, P. Ryzhakov, and B. Suárez. Particle-Based Methods: Fundamentals and Applications, chapter Advances in the Particle Finite Element Method (PFEM) for Solving Coupled Problems in Engineering, pages 1-49. Springer Netherlands, Dordrecht, 2011.

36. D Pelletier, A Fortin, and R Camarero. Are FEM solutions of incompressible flows really incompressible?(or how simple flows can cause headaches!). International Journal for Numerical in Fluids, 9:99-112, 1989.

37. J. M. Rodriguez, J. M. Carbonell, J. C. Cante, and X. Oliver. The particle finite element method (pfem) in thermo-mechanical problems. International Journal for Numerical Methods in Engineering, doi: 10.1002/nme.5186, 2016. 
38. P. Ryzhakov. An axisymetric pfem formulation for bottle forming simulation. Computational Particle Mechanics, DOI 10.1007/s40571-016-0114-7, 2016.

39. P. Ryzhakov, E. Oñate, and S. R. Idelsohn. Improving mass conservation in simulation of incompressible flows. International Journal of Numerical Methods in Engineering, 90:1435-1451, 2012.

40. P. Ryzhakov, R. Rossi, S. R. Idelsohn, and E. Oñate. A monolithic lagrangian approach for fluid-structure interaction problems. Computational Mechanics, 46:883-899, 2010.

41. A. Saalfeld. In Delaunay edge refinements, pages 33-36, Burnaby, 1991.

42. B. Tang, J. F. Li, and T. S. Wang. Some improvements on free surface simulation by the particle finite element method. International Journal for Numerical Methods in Fluids, 60 (9):1032-1054, 2009.

43. X. Zhang, K. Krabbenhoft, D. M. Pedroso, A. V. Lyamin, D. Sheng, M. Vicente da Silva, and D. Wang. Particle finite element analysis of large deformation and granular flow problems. Computer and Geotechnics, $54: 133-142,2013$

44. M. Zhu and M. H. Scott. Modeling fluid-structure interaction by the particle finite element method in opensees. Computers and Structures, 132:12-21, 2014.

45. O. C. Zienkiewicz, R. L. Taylor, and P. Nithiarasu. The Finite Element Method for Fluid Dynamics, Volume 3 (6th Ed.). Elsiever, Oxford, 2005. 